\title{
A DIMENSIONALLY CONTINUED POISSON SUMMATION FORMULA
}

\author{
NATHAN K. JOHNSON-MCDANIEL
}

\begin{abstract}
We generalize the standard Poisson summation formula for lattices so that it operates on the level of theta series, allowing us to introduce noninteger dimension parameters (using the dimensionally continued Fourier transform). When combined with one of the proofs of the Jacobi imaginary transformation of theta functions that does not use the Poisson summation formula, our proof of this generalized Poisson summation formula also provides a new proof of the standard Poisson summation formula for dimensions greater than 2 (with appropriate hypotheses on the function being summed). In general, our methods work to establish the (Voronoi) summation formulae associated with functions satisfying (modular) transformations of the Jacobi imaginary type by means of a density argument (as opposed to the usual Mellin transform approach). In particular, we construct a family of generalized theta series from Jacobi theta functions from which these summation formulae can be obtained. This family contains several families of modular forms, but is significantly more general than any of them. Our result also relaxes several of the hypotheses in the standard statements of these summation formulae. The density result we prove for Gaussians in the Schwartz space may be of independent interest.
\end{abstract}

\section{INTRODUCTION}

Consider a lattice $\Lambda \subset \mathbb{R}^{n}$ and a sufficiently well-behaved function $F: \mathbb{R}^{n} \rightarrow \mathbb{R}$. [Taking $F$ to belong to the Schwartz space $\mathscr{S}\left(\mathbb{R}^{n}\right)$ is sufficient, and is what we shall do in our later generalization.] The standard Poisson summation formula then says that

$$
\sum_{k \in \Lambda} F(k)=\frac{1}{\sqrt{\operatorname{det} \Lambda}} \sum_{p \in \Lambda^{*}} \tilde{F}(p)
$$

Here $\Lambda^{*}$ is the lattice dual to $\Lambda, \operatorname{det} \Lambda$ denotes the volume of a Voronoi cell of $\Lambda$, and

$$
\tilde{F}(p):=\int_{\mathbb{R}^{n}} F(x) e^{-2 \pi i x \cdot p} d x
$$

denotes the Fourier transform of $F$. (We use a tilde here so that we can reserve the circumflex for our more general, dimensionally continued Fourier transform.) We wish to construct a dimensionally continued version of this result.

This problem was originally inspired by a condensed matter physics investigation involving the dimensional continuation of electrostatic lattice sums, computed using the Ewald method (see, e.g., [20] for a modern exposition of this method), as described in [21]. However, the ensuing discussion is a purely mathematical offshoot of this investigation. For one thing, the results we were able to prove do not include the physically relevant case of a slowly decaying function, even though we have numerical evidence that the results still hold in this case. Nevertheless, the methods used here might also be applicable to the dimensional regularization of lattice sums: See [8] for an approach using zeta functions and the Mellin transform.

In addition to its use in performing lattice sums, the Poisson summation formula is a prominent tool in most other problems involving lattices, from point counting problems in number theory (discussed by, e.g., Miller and Schmid [26]), to the mathematical theory of diffraction-see, e.g., the review by Lagarias [23]. While we do not consider diffraction in this article, it is possible that our results might have some application to diffraction problems, or that results in the theory of diffraction might provide some further avenues for generalization of the results presented here. In particular, the quasicrystal summation formula given in Theorem 2.9 of Lagarias [23] seems to offer attractive possibilities for generalization. Additionally, Baake, Frettlöh, and Grimm [2] apply an integer dimension result that is very similar to our formula to a problem in diffraction.

If we specialize to the case where $F$ is a radial function, we can obtain a dimensionally continued Poisson summation formula in terms of the lattice's theta series—see Theorem 1 for the (particularly simple) version for $\mathbb{Z}^{d}(d \geq 1)$.

2010 Mathematics Subject Classification. Primary 42A38, 11F27, 11F30.

Key words and phrases. Summation formulae, Voronoi summation, theta functions, modular transformation. 
One can then generalize this result to a reasonably large family of theta series-like functions constructed out of linear combinations of products of powers of Jacobi theta functions; the generalization is given in Theorem 2 This family of generalized theta series includes all the theta series of lattices given in Chap. 4 of Conway and Sloane [10] (except for the general forms of those for the root lattice $A_{d}$ and its translates), as well as several families of modular forms (including all the classical modular forms of even weight), as discussed by Rankin [31], and more compactly stated as Theorem 12 in Pache [30]. However, the family we consider is considerably more general, since we only require integer powers to reproduce the aforementioned theta series from Conway and Sloane and the families of modular forms from Rankin, while here the powers can be arbitrary nonnegative real numbers.

This relation between modular transformations and summation formulae is not new. In fact, much of the basic result we obtain was first given by Bochner [5]. [The summation formula is stated in Bochner's Eq. (80) in an integral form and in his Eq. (129) using sums; a nice form of sufficient hypotheses on the function being summed are given in Bochner's Theorem 9. Also see Theorem 4.9.1 in [6] for a more streamlined presentation of the summation formula (using sums), though with some obvious typos, and without a statement of the hypotheses concerning the function being summed.] Our method of proof bears some similarities to Bochner's, though Bochner uses Fourier-Laplace transforms as the basic analytic tool, while we use a density result. (However, Bochner's method allows for weaker hypotheses.) Our overall focus is also somewhat different, in that we are concerned with the summation formulae derived from a specific family of generalized theta series, while Bochner only considers a general modular relation. As far as we know, this specific construction of a family of dimensionally continued Poisson summation formulae has not been considered previously. While Guinand gives an explicit analogue to our Theorem 1 in Sec. 10.5 of [15], this is only stated for integer dimensions, even though Guinand cites Bochner as a reference for the formula. (Guinand also cites his own general summation formula from [14], which is only given for integer dimensions.) Note that we only became aware of the work of Bochner and Guinand in the process of publication, through the reference to [15] in the review by Lagarias [23] that the referee suggested to us.

In more recent work, the relation has reached its most refined form in the association between automorphic forms and Voronoi summation formulae (see, e.g., Miller and Schmid [26] for a review of recent work). However, work on this correspondence first arose in the context of transformations related to the functional equation for the zeta function, inspired by a question from Voronoi on analogues of the Poisson summation formula—see, e.g., [36] and references therein. (We call particular attention to the work of Ferrar [13]; see Theorem 10.2.17 in Cohen [7] for a more modern discussion of a very similar result.) Additionally, Baake, Frettlöh, and Grimm [2] give a (distributional) radial Poisson summation formula in their Theorem 3 in a form that is very similar to our dimensionally continued form. However, they do not show how to dimensionally continue the lattice (or, indeed, mention theta functions explicitly), and their proof (which relies on the standard Poisson summation formula) only holds for integer dimensions. There are also discussions of similar formulae - these derived from modular transformations - at the beginning of Chap. 4 of Iwaniec and Kowalski [18], and in Sec. 10.2 of Huxley [17]—what Huxley terms the Wilton summation formula. These formulae are presented in what appears to be a dimensionally continued form, though their hypotheses assume integer dimensions. Regardless, the summation formula in Iwaniec and Kowalski and Huxley's Wilton summation formula are derived from cusp forms, while our result (in the language of modular forms) does not require the vanishing of the constant term in the form's Fourier series (the defining characteristic of a cusp form).

We make considerable use of various special functions in the following discussion, so we provide references for the conventions we use. The dimensionally continued theta series for which our summation formula holds will all be constructed out of Jacobi theta functions. An appropriate introduction to these functions for our purposes is given in Chap. 4 of Conway and Sloane [10] [though there is an important difference in our notation, as discussed below Eq. [3]]. We shall also encounter the confluent hypergeometric limit function and the Hermite functions (plus a brief appearance by a Bessel function). These are discussed in an appropriate way by Andrews, Askey, and Roy [1] (though we slightly streamline their general hypergeometric function notation, since we only consider the confluent hypergeometric limit function). In general, the Wolfram Functions Site [41] is a good resource for information about the various special functions we employ.

Additionally, we shall need the apparatus of tempered distributions introduced by L. Schwartz [34]. A convenient overview of the properties we need is given in Sec. V.3 of Reed and Simon [32]. (The Appendix to that section also provides another exposition of the $N$-representation we use.)

\section{INGREDIENTS}


2.1. Theta series. Here we recall various facts about theta series and theta functions that we shall need for the rest of our discussion, following Chap. 4 of Conway and Sloane [10]. The theta series of a lattice $\Lambda$ is defined by

$$
\Theta_{\Lambda}(q):=\sum_{k \in \Lambda} q^{|k|^{2}}
$$

(This is often treated as a formal series, but converges for $q \in \mathbb{C},|q|<1$.) The utility of the theta series stems from the fact that the coefficient of $q^{l}$ in the expansion of $\Theta_{\Lambda}(q)$ in powers of $q$ gives the number of points in the intersection of the lattice and a sphere of radius $\sqrt{l}$ centred at the origin. Thus, if we write

$$
\Theta_{\Lambda}(q)=: \sum_{l=0}^{\infty} N_{l} q^{A_{l}}
$$

then

$$
\sum_{k \in \Lambda} f(|k|)=\sum_{l=0}^{\infty} N_{l} f\left(\sqrt{A_{l}}\right)
$$

[Nota bene: We have written the radial function $F$ as $f(|\cdot|)$, and shall only consider these radial parts in the sequel.] Examples of dimensionally continued theta series for families of lattices include the $d$-dimensional cubic lattice $\mathbb{Z}^{d}$, with $\Theta_{\mathbb{Z}^{d}}(q)=\vartheta_{3}^{d}(q)$, and the root lattice $D^{d}$, with $\Theta_{D^{d}}(q)=\left[\vartheta_{3}^{d}(q)+\vartheta_{4}^{d}(q)\right] / 2$. (See Chap. 4 in Conway and Sloane [10] for further examples.) Here

$$
\vartheta_{2}(q):=2 q^{1 / 4} \sum_{l=1}^{\infty} q^{l^{2}-l}, \quad \vartheta_{3}(q):=1+2 \sum_{l=1}^{\infty} q^{l^{2}}, \quad \vartheta_{4}(q):=1+2 \sum_{l=1}^{\infty}(-q)^{l^{2}}
$$

are Jacobi theta functions (where $\vartheta_{2}$ is defined for future use).

Nota bene: It is often customary to take theta functions and theta series to be functions of a complex variable $z$, instead of the nome $q=e^{i \pi z}$ that we have used here. We have chosen to regard the nome as fundamental since we are primarily interested in the expansions of these functions in powers of $q$. However, when discussing transformations of these functions, it is considerably more convenient to regard them as functions of $z$. On the few occasions where we do this, we shall use an overbar to denote the difference, e.g., $\bar{\Theta}_{\Lambda}(z):=\Theta_{\Lambda}\left(e^{i \pi z}\right)$. (In the literature on summation formulae derived from automorphic forms, one thinks of our expansions of $\Theta_{\Lambda}$ in $q$ as the Fourier coefficients of $\bar{\Theta}_{\Lambda}$.)

Since the Poisson summation formula involves the dual lattice, we need to know how to obtain its theta series. This is given by the Jacobi formula [Eq. (19) in Chap. 4 of Conway and Sloane [10]], which states that

$$
\bar{\Theta}_{\Lambda^{*}}(z)=\sqrt{\operatorname{det} \Lambda}(i / z)^{d / 2} \bar{\Theta}_{\Lambda}(-1 / z),
$$

where $d$ is the dimension of the lattice. The Jacobi formula is typically proved using the Poisson summation formula [see, e.g., the discussion leading up to our Eq. (8)]. However, all we need in our discussion is the intimately related Jacobi imaginary transformation of the Jacobi theta functions (also known as the modular identity or reciprocity formula for the theta functions), i.e.,

$$
\bar{\vartheta}_{2}(-1 / z)=(z / i)^{1 / 2} \bar{\vartheta}_{4}(z), \quad \bar{\vartheta}_{3}(-1 / z)=(z / i)^{1 / 2} \bar{\vartheta}_{3}(z) .
$$

(The first of these is also true with the labels 2 and 4 switched.) The standard proof of these identities is a direct application of the Poisson summation formula, but there are alternative proofs that are independent of it. For instance, one such proof is given in Sec. 21.51 of Whittaker and Watson [40], while Bellman's text [4] discusses several otherssee, in particular, Sec. 30 for Polya's derivation-in addition to the standard Poisson summation version (in Sec. 9). Our discussion will thus be independent of the standard Poisson summation formula (with the exception of a brief appeal to establish Theorem 1 for $d=1$ ).

2.2. The dimensionally continued Fourier transform. We also need to dimensionally continue the Fourier transform. Stein and Weiss give a dimensionally continued version of the Fourier transform for radial functions in Theorem 3.3 of Chap. IV of [37], viz.,

$$
\begin{aligned}
\hat{f}(p) & :=2 \pi p^{-(d-2) / 2} \int_{0}^{\infty} f(r) J_{(d-2) / 2}(2 \pi p r) r^{d / 2} d r \\
& =\frac{2 \pi^{d / 2}}{\Gamma(d / 2)} \int_{0}^{\infty} f(r)_{0} F_{1}\left(d / 2 ;-\pi^{2} p^{2} r^{2}\right) r^{d-1} d r .
\end{aligned}
$$


(This reduces to the standard Fourier transform for a radial function when $d \in \mathbb{N}$.) Here the first equality gives the expression from Stein and Weiss ( $J_{k}$ is a Bessel function) and the second gives an equivalent (perhaps slightly neater) expression in terms of the confluent hypergeometric limit function ${ }_{0} F_{1}$. The hypergeometric expression has the advantage of only involving one appearance of $p$ (and being manifestly regular at $p=0$ for all $d \geq 1$ ), in addition to showing the $d$-dimensional polar coordinate measure for radial functions explicitly. We shall thus use the hypergeometric expression exclusively in the sequel. (One can obtain the hypergeometric expression using the Stein and Weiss derivation-the only difference is that one uses a different special function to evaluate the final integral 1 )

For this expression to be well-defined, it is sufficient to take $d \geq 1$ : One assumes $d>1$ when using integral representations to express the result in terms of either of the two given special functions, and can also check that the integral is convergent for all $p \in \mathbb{R}$ in that case, provided that $f \in L^{1}\left(\mathbb{R}_{+}\right)$. Additionally, Eq. (6) reduces to the expected expression for $d=1$ [using $J_{-1 / 2}(z)=\sqrt{2 / \pi z} \cos z$ or ${ }_{0} F_{1}(1 / 2 ;-z)=\cos (2 \sqrt{z})$ ] ${ }^{2}$ (Stein and Weiss restrict to $d \geq 2$ so that the integral they use in their derivation is well-defined, since they are only considering integer dimensions.) This restriction to $d>1$ is necessary for other parts of our discussion, though we have numerical evidence that it can be relaxed.

The following result is central to understanding why this dimensionally continued Fourier transform agrees with the dimensional continuation of the theta series.

Lemma 1. For $d \geq 1$, the dimensionally continued Fourier transform (for radial functions) defined in Eq. (6) takes a Gaussian $\mathcal{G}_{\alpha}(r):=e^{-\alpha r^{2}}, \operatorname{Re} \alpha>0$ to another Gaussian, $\hat{\mathcal{G}}_{\alpha}(p)=(\pi / \alpha)^{d / 2} e^{-\pi^{2} p^{2} / \alpha}$.

Remark. Intuitively, this result follows from dimensionally continuing the well-known integer dimension result. We should get the same result from direct calculation using Eq. (6) since that expression was obtained using the same dimensional continuation procedure.

Proof. The case $d=1$ is classical. For $d>1$, we use ${ }_{0} F_{1}$ 's defining series,

$$
{ }_{0} F_{1}\left(d / 2 ;-\pi^{2} p^{2} r^{2}\right)=\sum_{n=0}^{\infty} \frac{\left(-\pi^{2} p^{2} r^{2}\right)^{n}}{(d / 2)_{n} n !}
$$

$\left[(\cdot)_{n}\right.$ denotes the Pochhammer symbol $]$ and integrate term-by-term, evaluating each integral using the gamma function 3 The resulting series is the Maclaurin series for the expression we gave for $\hat{\mathcal{G}}_{\alpha}$. The term-by-term integration is justified by the Lebesgue dominated convergence theorem. To see this, we use the same integral representation for ${ }_{0} F_{1}$ used in the derivation of Eq. [6], which gives, for any $N \in \mathbb{N}$,

$$
\left|\sum_{n=0}^{N} \frac{\left(-\pi^{2} p^{2} r^{2}\right)^{n}}{(d / 2)_{n} n !}\right| \leq{ }_{0} F_{1}\left(d / 2 ; \pi^{2} p^{2} r^{2}\right) \leq K \cosh (2 \pi p r) \int_{0}^{1}\left(1-t^{2}\right)^{(d-3) / 2} d t,
$$

where $K>0$ is a constant 4 This allows us to apply the dominated convergence theorem, since the integral in the final term is finite for $d>1$ and $\int_{0}^{\infty} \cosh (2 \pi p r)\left|e^{-\alpha r^{2}}\right| d r$ is finite for $\operatorname{Re} \alpha>0$.

Remark. The importance of this result to our discussion comes in its use in obtaining the integer dimension Jacobi transformation formula (and thus also the Jacobi imaginary transformations of the Jacobi theta functions) via the standard Poisson summation formula: For a lattice $\Lambda$ of dimension $n \in \mathbb{N}$, we have (taking $\operatorname{Im} z>0$ so that everything converges)

$$
\bar{\Theta}_{\Lambda}(z):=\sum_{k \in \Lambda} e^{i \pi z|k|^{2}}=\frac{1}{\sqrt{\operatorname{det} \Lambda}}\left(\frac{i}{z}\right)^{n / 2} \sum_{p \in \Lambda^{*}} e^{-i \pi|p|^{2} / z}=\frac{1}{\sqrt{\operatorname{det} \Lambda}}\left(\frac{i}{z}\right)^{n / 2} \bar{\Theta}_{\Lambda^{*}}(-1 / z),
$$

which can be written as

$$
\bar{\Theta}_{\Lambda^{*}}(z)=\sqrt{\operatorname{det} \Lambda}(i / z)^{n / 2} \bar{\Theta}_{\Lambda}(-1 / z)
$$

\footnotetext{
${ }^{1}$ The integral representation for ${ }_{0} F_{1}$ we used is 07.17.07.0004.01 on the Wolfram Functions Site [41].

${ }^{2}$ These identities are 03.01.03.0004.01 and 07.17.03.0037.01, respectively, on the Wolfram Functions Site [41].

${ }^{3}$ The Maclaurin series for ${ }_{0} F_{1}$ is 07.17 .02 .0001 .01 on the Wolfram Functions Site [41].

${ }^{4}$ Nota bene: We denote the set of positive integers by $\mathbb{N}$, and the set of nonnegative integers by $\mathbb{N}_{0}$.
} 
the Jacobi transformation formula. We thus expect that the dimensionally continued dual theta series that we obtain using this formula will agree with the dimensionally continued Fourier transform to give a dimensionally continued Poisson summation formula.

\section{The Dimensionally CONTINUED POISSON SUMMATION FORMULA FOR $\mathbb{Z}^{d}$}

With these results in hand, we can thus write Eq. (1) [for a radial function $F=: f(|\cdot|)$ ] as

$$
\sum_{l=0}^{\infty} N_{l} f\left(\sqrt{A_{l}}\right)=\frac{1}{\sqrt{\operatorname{det} \Lambda}} \sum_{l=0}^{\infty} N_{l}^{*} \hat{f}\left(\sqrt{A_{l}^{*}}\right),
$$

where the starred quantities come from writing the theta series of $\Lambda^{*}$ in the power series form given by Eq. (2), and we calculate $\hat{f}$ by taking the dimension parameter $d$ to be the dimension of the lattice. (As we shall see later, what is important is that the $d$ one uses here is the same $d$ that appears in the Jacobi transformation formula.) It is clear that this equality holds when $d \in \mathbb{N}$, by the standard Poisson summation formula. What is perhaps surprising is that the equality still holds for, e.g., $\Lambda=\mathbb{Z}^{d}$, with $d \in \mathbb{R}(d \geq 1)$. We shall first prove the result for this simple case ( $\mathbb{Z}^{d}$ is self-dual, $\operatorname{det} \mathbb{Z}^{d}=1$, and $A_{l}=l$ ), where it becomes

Theorem 1. If $f \in \mathscr{S}^{\mathrm{E}}(\mathbb{R})$ (i.e., $f$ is an even Schwartz function) and $d \geq 1$, then

$$
\sum_{l=0}^{\infty} N_{l} f(\sqrt{l})=\sum_{l=0}^{\infty} N_{l} \hat{f}(\sqrt{l})
$$

where the $N_{l}$ are given by the power series expansion of the theta series of $\mathbb{Z}^{d}$, viz.,

$$
\Theta(q)=\vartheta_{3}^{d}(q)=\left[1+2 \sum_{k=1}^{\infty} q^{k^{2}}\right]^{d}=: \sum_{l=0}^{\infty} N_{l} q^{l},
$$

and $\hat{f}$ is computed using Eq. (6).

However, the simplifications are primarily notational. As we shall see in the discussion in Sec.6 the proof works with minimal modifications for a much larger class of $\Theta$ s, including functions that cannot be the theta series of a lattice (even though they have an integer dimension parameter).

Remark. The restriction that $f$ be an even function should not be surprising: In integer dimensions, it corresponds to the lack of a cusp at the origin for the full radial function $F=f(|\cdot|)$. Moreover, as Miller and Schmid note [26], the standard one-dimensional Poisson summation formula is a trivial $0=0$ for odd functions.

\section{A SCHWARTZ SPACE DENSITY RESUlT}

Since the proof proceeds by noting that the desired formula holds almost trivially for the Gaussians from Lemma 1 and then extends to an interesting set of functions [viz., $\left.\mathscr{S}^{\mathrm{E}}(\mathbb{R})\right]$ by density, we start by establishing the requisite density result.

Lemma 2. $\overline{\operatorname{Span}\left\{x \mapsto e^{-\alpha x^{2}} \mid \alpha>0\right\}} \stackrel{\mathscr{S}(\mathbb{R})}{=} \mathscr{S}^{\mathrm{E}}(\mathbb{R})$ [i.e., the Schwartz space closure of the given family of Gaussians is all the even Schwartz functions].

Proof. We shall prove this by showing that

$$
\mathcal{X}:=\operatorname{Span}\left\{x \mapsto e^{-\alpha x^{2}} \mid \alpha>0\right\}+\operatorname{Span}\left\{x \mapsto x e^{-\alpha x^{2}} \mid \alpha>0\right\}
$$

is dense in $\mathscr{S}(\mathbb{R})$, so its even part, $\operatorname{Span}\left\{x \mapsto e^{-\alpha x^{2}} \mid \alpha>0\right\}$, is thus dense in $\mathscr{S}^{\mathrm{E}}(\mathbb{R})$. We shall use Corollary IV.3.14 from Conway's text [9], which states that a linear manifold (here $\mathcal{X}$ ) is dense in a locally convex topological vector space $[$ here $\mathscr{S}(\mathbb{R})]$ if and only if the only element of the dual of the topological vector space that vanishes on all elements of the linear manifold is the zero element.

It is most convenient to proceed by identifying $\mathscr{S}(\mathbb{R})$ with a sequence space, following Simon [35]. (There is an alternative presentation of these results in the Appendix to Sec. V of Reed and Simon [32].) Here the sequence space is given by the coefficients of the Hermite function expansion of elements of $\mathscr{S}(\mathbb{R})$, and provides a particularly nice characterization of the tempered distributions [the elements of $\mathscr{S}^{\prime}(\mathbb{R})$, the dual of $\mathscr{S}(\mathbb{R})$ ]. Namely, if $a_{n}$ are 
the Hermite coefficients of $f \in \mathscr{S}(\mathbb{R})$ [i.e., $a_{n}:=\int_{\mathbb{R}} f(x) h_{n}(x) d x$, where $h_{n}$ is the $n$th Hermite function], then $\varphi \in \mathscr{S}^{\prime}(\mathbb{R})$ can be written as $\varphi(f)=\sum_{n=0}^{\infty} c_{n} a_{n}$, where $c_{n}$ are the Hermite coefficients of $\varphi$, with $\left|c_{n}\right| \leq C(1+n)^{m}$ for some $C, m>0$. (This is Theorem 3 in Simon [35].) Note that Simon defines the Hermite functions to be $L^{2}$ normalized, so, we have, from the first equation in Sec. 2 of Simon

$$
h_{n}(x):=\frac{e^{-x^{2} / 2}}{\sqrt{\pi^{1 / 2} 2^{n} n !}} H_{n}(x), \quad H_{n}(x):=(-1)^{n} e^{x^{2}} \frac{d^{n}}{d x^{n}} e^{-x^{2}},
$$

where the $H_{n}$ are the Hermite polynomials, with generating function 9

$$
\sum_{n=0}^{\infty} H_{n}(x) \frac{t^{n}}{n !}=e^{2 t x-t^{2}} .
$$

We can now use this generating function to show that the Hermite coefficients of $x \mapsto e^{-\alpha x^{2}}$ are given by

$$
a_{n}=\left.\mathcal{N}_{n} \frac{d^{n}}{d t^{n}}\left[\int_{\mathbb{R}} e^{-\left(\alpha x^{2}+x^{2} / 2-2 t x+t^{2}\right)} d x\right]\right|_{t=0}=\left.\mathcal{N}_{n} \frac{d^{n}}{d t^{n}}\left[\sqrt{\pi \beta} e^{(\beta-1) t^{2}}\right]\right|_{t=0},
$$

where $\mathcal{N}_{n}:=\left(\pi^{1 / 2} 2^{n} n !\right)^{-1 / 2}$ is the Hermite functions' normalization factor and $\beta:=1 /(\alpha+1 / 2)$. We thus have $a_{2 n}=\mathcal{N}_{2 n}(\pi / \beta)^{1 / 2}(\beta-1)^{n} / n !, a_{2 n+1}=0$, by the series expansion of the exponential. [We used Lemma 2.2 in Chap. VIII of Lang [24] to interchange differentiation and integration. We only need to consider the case where $t$ lies in some neighbourhood of 0 , so the $t$-derivatives of the integrand are each bounded by a polynomial in $x$ times a Gaussian in $x$ (for all $t$ in the neighbourhood), and those functions of $x$ are integrable over $\mathbb{R}$.] Similarly, the Hermite coefficients of $x \mapsto x e^{-\alpha x^{2}}$ are $b_{2 n}=0$ and $b_{2 n+1}=\mathcal{N}_{2 n+1}\left(\pi / \beta^{3}\right)^{1 / 2}(\beta-1)^{n} / n$ !. Thus, we consider

$$
E_{\beta, \pm}(x):=(\beta / \pi)^{1 / 2} e^{-\alpha x^{2}} \pm\left(\beta^{3} / \pi\right)^{1 / 2} x e^{-\alpha x^{2}},
$$

which has Hermite coefficients of $( \pm 1)^{n} \mathcal{N}_{n}(\beta-1)^{\lfloor n / 2\rfloor} /\lfloor n / 2\rfloor$ !, where $\lfloor\cdot\rfloor$ denotes the greatest integer less than or equal to its argument.

Now, for any $\varphi \in \mathscr{S}^{\prime}(\mathbb{R}), \mathcal{E}_{ \pm}(\beta):=\varphi\left(E_{\beta, \pm}\right)$ is a holomorphic function of $\beta$. To see this, we note that

$$
\mathcal{E}_{ \pm}(\beta)=\sum_{n=0}^{\infty}( \pm 1)^{n} c_{n} \mathcal{N}_{n} \frac{(\beta-1)^{\lfloor n / 2\rfloor}}{\lfloor n / 2\rfloor !}=\sum_{n=0}^{\infty}\left(\mathcal{N}_{2 n} c_{2 n} \pm \mathcal{N}_{2 n+1} c_{2 n+1}\right) \frac{(\beta-1)^{n}}{n !}
$$

where $c_{n}$ are the Hermite coefficients of $\varphi$. Since the $c_{n}$ are bounded by a polynomial in $n$, the series converges for all $\beta \in \mathbb{C}$, giving holomorphy. Thus, if $\mathcal{E}_{ \pm}(\beta)=0$ for all $\beta$ in an interval (as is the case here), then all of $\mathcal{E}_{ \pm}$'s power series coefficients are zero. Applying this result to the two choices of sign, we obtain (since the $\mathcal{N}_{n}$ are never zero) $c_{n}=0 \forall n \in \mathbb{N}_{0} \Rightarrow \varphi \equiv 0$, which thus proves the lemma.

Remark. This result may be of wider applicability, particularly in harmonic analysis, due to the ubiquity of the Gaussian. We thus note that the proof of the lemma shows that $\alpha$ need merely belong to some subset of the right half-plane with an accumulation point to guarantee density. One could have also proved this result more abstractly (and without recourse to the Hermite expansion) by a slightly indirect application of the Stone-Weierstrass theorem, though the basic Hahn-Banach argument (contained in the Corollary from Conway we use) remains the same

\section{Proof of Theorem 1}

We first note that Eq. (9) is clearly true for $d=1$ (indeed, $d \in \mathbb{N}$ ) by the standard Poisson summation formula for lattices (applied to $\mathbb{Z}^{d}$ ). To prove the result for $d>1$, we shall first establish that it holds for the Gaussians from Lemma 1 and then show that the equality still holds in the limit in the Schwartz space topology. The control afforded by demanding convergence in the Schwartz space makes this quite straightforward. The primary result that needs to be shown is that two functions that are $\epsilon$-close in the Schwartz space topology have dimensionally continued Fourier

\footnotetext{
${ }^{5}$ Nota bene: Simon defines the $h_{n}$ without the factor of $(-1)^{n}$ (that here comes from our $H_{n}$ ). We have included the $(-1)^{n}$ for notational simplicity (since we use the standard convention for the Hermite polynomials). This does not have any effect on Simon's Theorem 3 , since it simply amounts to a sign change of the odd Hermite coefficients.

${ }^{6}$ This is 05.01.11.0001.01 on the Wolfram Functions Site [41].

${ }^{7}$ Personal communication from John Roe.
} 
transforms that are $C \epsilon$-close in a given Schwartz space seminorm (where the constant $C$ depends on the seminorm under consideration, as well as $d$ ).

To show that Eq. (9) holds when $f=\mathcal{G}_{\alpha}$, we first consider the left-hand side and note that

$$
\sum_{l=0}^{\infty} N_{l} e^{-\alpha l}=\Theta\left(e^{-\alpha}\right) .
$$

Convergence is guaranteed because $\Theta$ is analytic inside the unit disk. [To see that $\Theta$ is analytic inside the unit disk, note that $\vartheta_{3}$ is analytic there, and, moreover, nonzero, so its $d$ th power is analytic, as well. It is easiest to see that $\vartheta_{3}$ is nonzero inside the unit disk from its infinite product expansion, given in, e.g., Eq. (35) in Chap. 4 of Conway and Sloane [10].] Using Lemma1, the right-hand side of Eq. 90 becomes

$$
\left(\frac{\pi}{\alpha}\right)^{d / 2} \sum_{l=0}^{\infty} N_{l} e^{-\pi^{2} l / \alpha}=\left(\frac{\pi}{\alpha}\right)^{d / 2} \Theta\left(e^{-\pi^{2} / \alpha}\right) .
$$

Now, the Jacobi imaginary transformation for $\vartheta_{3}$ [Eq. (5]] implies that $(\pi / \alpha)^{d / 2} \Theta\left(e^{-\pi^{2} / \alpha}\right)=\Theta\left(e^{-\alpha}\right)$, so we have thus established the result for $\mathcal{G}_{\alpha}$.

We shall now show that this equality continues to hold in the limit. The equality is clearly true for any finite linear combination of the Gaussians from Lemma 1 so we use Lemma 2 to approximate an arbitrary $f \in \mathscr{S}^{\mathrm{E}}(\mathbb{R})$ by a finite linear combination of these Gaussians, $g$. Specifically, we have $\|f-g\|_{n, m}<\epsilon \forall n, m \in \mathbb{N}_{0}$, where $\|f\|_{n, m}:=\sup _{x \in \mathbb{R}}\left|x^{n} f^{(m)}(x)\right|$ is the family of seminorms that gives the Schwartz space topology. (We denote the $m$ th derivative of $f$ by $f^{(m)}$.) We wish to bound the difference between the two sides of Eq. (9) by a constant times $\epsilon$. We have

$$
\left|\sum_{l=0}^{\infty} N_{l} f(\sqrt{l})-\sum_{l=0}^{\infty} N_{l} \hat{f}(\sqrt{l})\right| \leq\left|\sum_{l=0}^{\infty} N_{l}(f-g)(\sqrt{l})\right|+\left|\sum_{l=0}^{\infty} N_{l}(\hat{f}-\hat{g})(\sqrt{l})\right|,
$$

where we used the fact that the dimensionally continued Poisson summation formula holds for $g$, along with the triangle inequality. We can bound the two sums on the right-hand side by constants times $\epsilon$ using the assumption about the closeness of $f$ to $g$ in the Schwartz space topology and the fact that $N_{l}$ grows at most polynomially with $l$. The latter fact also shows that the two sums on the left converge for $f \in \mathscr{S}(\mathbb{R})$.

\subsection{Bounds on the growth of $N_{l}$ and on the right-hand side of Eq. (13).}

Lemma 3. The $N_{l}$ defined in Eq. (10) are polynomially bounded. Specifically, we have

$$
\left|N_{l}\right| \leq 2^{d}(1+d / l)^{l}(1+l / d)^{d} \leq C_{d} l^{d},
$$

where $C_{d}>0$ is some constant (and the second inequality only holds for $l \geq 1$ ).

Proof. Recalling that $\Theta$ is analytic inside the unit disk, we can apply Cauchy's integral formula to the contour $C_{R}$, a circle of radius $R \in(0,1)$, centered at the origin (and oriented counterclockwise), to obtain

$$
\left|N_{l}\right|=\left|\frac{1}{2 \pi i} \int_{\mathrm{C}_{R}} \frac{\vartheta_{3}^{d}(z)}{z^{l+1}} d z\right|=\frac{1}{2 \pi}\left|\int_{0}^{2 \pi} \frac{\vartheta_{3}^{d}\left(R e^{i \theta}\right)}{R^{l} e^{i l \theta}} d \theta\right| \leq \frac{2^{d}}{R^{l}(1-R)^{d}} .
$$

Here we have used $\left|\vartheta_{3}(q)\right| \leq 2 /(1-|q|)$ (for $|q|<1$, obtained using the geometric series). The right-hand side attains its minimum [for $R \in(0,1)]$ at $R=l /(l+d)$, so we have the desired result. To obtain the second inequality, we use the fact that $(1+1 / r)^{r}<e$ for $r>0$.

Remark. While this bound is easy to obtain and is all that is necessary for our purposes, it is by no means optimal. For instance, for integer $d$, we can apply the Hecke bound for modular forms (e.g., Theorem 4.5.3 in Rankin [31]) to conclude that $N_{l}=O\left(l^{d / 2}\right)$. (Other of Rankin's results—stated as Theorem 12 in Pache [30] - show that the Hecke bound stated by Rankin is applicable to $\vartheta_{3}^{d}$ for $d \in \mathbb{N}$.)

If we write $h:=f-g$, then this bound implies that $\left|N_{l} h(\sqrt{l})\right| \leq C_{d} l^{d}|h(\sqrt{l})| \leq \epsilon C_{d} / l^{2}$ (for $l \geq 1$ ), where the second inequality follows from the fact that $h$ is $\epsilon$-close to 0 in the Schwartz space topology. [Explicitly, we have $\left|x^{2 d+4} h(x)\right| \leq \epsilon \forall x>1 \Rightarrow l^{d}|h(\sqrt{l})| \leq \epsilon / l^{2} \forall l \in \mathbb{N}$. The first inequality comes from noticing that for any $\gamma \geq 0$, we have $\left|x^{\gamma} h(x)\right| \leq\left|x^{\lceil\gamma\rceil} h(x)\right| \leq \epsilon$ for $x \geq 1$, where $\lceil\cdot\rceil$ denotes the smallest integer greater than or equal to its 
argument.] We shall show that $\left|p^{2 n} \hat{h}(p)\right| \leq K_{d} \epsilon \forall n \in \mathbb{N}, p \in \mathbb{R}$ (where $K_{d}$ is some $n$-dependent constant), so we have $\left|p^{2 d+4} \hat{h}(p)\right| \leq K_{d} \in \forall p \in \mathbb{R}$. We can thus apply the same argument to the second sum and hence bound both sums by constants times $\epsilon$ ( since $\sum_{l=1}^{\infty} l^{-2}$ is finite), showing that the dimensionally continued Poisson summation formula is true in the limit [since we will have shown that the right-hand side of Eq. (13) is bounded by a constant times $\epsilon]$.

5.2. Bound on $\left|p^{2 n} \hat{h}(p)\right|$. To prove the bound on $\left|p^{2 n} \hat{h}(p)\right|$, we first dimensionally continue some standard Fourier results.

Lemma 4. If we define the d-dimensional Laplacian for radial functions by

$$
\triangle_{d} f(r):=f^{\prime \prime}(r)+\frac{d-1}{r} f^{\prime}(r)
$$

then, for $d>1$,

i) $\mathcal{F}_{p}(r):={ }_{0} F_{1}\left(d / 2 ;-\pi^{2} p^{2} r^{2}\right)$ satisfies $\triangle_{d} \mathcal{F}_{p}=-4 \pi^{2} p^{2} \mathcal{F}_{p}$, so

ii) $\widehat{\triangle_{d}^{n f}}(p)=(-1)^{n}(2 \pi p)^{2 n} \hat{f}(p)$ for $f \in \mathscr{S}(\mathbb{R})$.

Proof. Part $i$ follows from the fact that $y_{a}(r):={ }_{0} F_{1}(a ; r)$ satisfies $r y_{a}^{\prime \prime}(r)+a y_{a}^{\prime}(r)=y_{a}(r){ }^{8}$ [Alternatively, it can be obtained by direct calculation using Eq. (7), justifying term-by-term differentiation using analyticity.] Part $i i$ is then obtained by induction, applying Eq. (6) to $\triangle_{d}^{n-1} f$ and integrating by parts twice. [The boundary terms at infinity vanish because $f \in \mathscr{S}(\mathbb{R})$; those at 0 vanish because $d>1$ (or cancel amongst themselves).]

We can thus write $\left|p^{2 n} \hat{h}(p)\right|=(2 \pi)^{-2 n}\left|\widehat{\triangle_{d}^{n} h}(p)\right|$. Then, since we shall show below that $\left|r^{k} \triangle_{d}^{n} h(r)\right| \leq \mathcal{D} \epsilon$, where $\mathcal{D}$ is some ( $n$ - and $d$-dependent constant), we obtain [using Eq. (6) and the fact that ${ }_{0} F_{1}(a ; r)$ is a bounded function of $r$, as was seen in the proof of Lemma 1

$$
\begin{aligned}
\left|p^{2 n} \hat{h}(p)\right| & \leq \mathcal{C} \int_{0}^{\infty}\left|\triangle_{d}^{n} h(r)\right| r^{d-1} d r \\
& \leq \mathcal{C}\left[\int_{0}^{1}\left|\triangle_{d}^{n} h(r)\right| d r+\int_{1}^{\infty}\left|\triangle_{d}^{n} h(r)\right| r^{d-1} d r\right] \\
& \leq \mathcal{C D}\left[1+\int_{1}^{\infty} r^{d-1-s} d r\right] \epsilon,
\end{aligned}
$$

where $\mathcal{C}>0$ is some ( $n$ - and $d$-dependent) constant and we used $\left|r^{k} \triangle_{d}^{n} h(r)\right| \leq \mathcal{D} \epsilon$ with $k=0$ and $k=s$. We can choose $s>d$, so the integral in the final term is finite, thus giving the desired result.

5.3. Bound on $\left|r^{k} \triangle_{d}^{n} h(r)\right|$. To see that $\left|r^{k} \triangle_{d}^{n} h(r)\right|$ is bounded by some ( $n$ - and $d$-dependent) constant (called $\mathcal{D}$ above), we first note that we can use induction to write

$$
\triangle_{d}^{n} h(r)=\sum_{j=1}^{2 n} a_{j} \frac{h^{(j)}(r)}{r^{2 n-j}}
$$

for some ( $n$ - and $d$-dependent) constants $a_{j}$ (and an arbitrary differentiable $h$ ). Thus, for $|r| \geq 1$, we have $\left|r^{k} \triangle_{d}^{n} h(r)\right| \leq$ $\epsilon \sum_{j=1}^{2 n} a_{j}$. For $|r|<1$, matters are considerably more subtle, and we have to rely on the fact that $h$ is even to see that $\triangle_{d}^{n} h$ remains bounded at the origin. The argument goes as follows: We write $h=\mathcal{P}+\mathcal{R}$, where $\mathcal{P}$ is $h$ 's $(2 n)$ th-degree Maclaurin polynomial (necessarily even, since $h$ is) and $\mathcal{R}$ is the associated remainder. We then have $\left|r^{k} \triangle_{d}^{n} h(r)\right| \leq\left|r^{k} \triangle_{d}^{n} \mathcal{P}(r)\right|+\left|r^{k} \triangle_{d}^{n} \mathcal{R}(r)\right|$. Since $\triangle_{d}^{n}$ maps even polynomials to even polynomials [as can be seen from Eq. [15] ], $\left|r^{k} \triangle_{d}^{n} \mathcal{P}(r)\right|$ is bounded by a $(k-, n$-, and $d$-dependent) constant times $\epsilon$ for $|r| \leq 1$. [Since the coefficients of $\mathcal{P}$ are given by derivatives of $h$, they are bounded by constants times $\epsilon$, by hypothesis.] To deal with $\left|r^{k} \triangle_{d}^{n} \mathcal{R}(r)\right|$, we first need to establish an identity for derivatives of $\mathcal{R}$, viz., (for $j \leq 2 n$ )

$$
\mathcal{R}^{(j)}(r)=\frac{h^{(2 n+1)}\left(\xi_{j}\right)}{(2 n+1-j) !} r^{2 n+1-j},
$$

\footnotetext{
${ }^{8}$ This differential equation for ${ }_{0} F_{1}$ is 07.17 .13 .0003 .01 on the Wolfram Functions Site [41].
} 
for some $\xi_{j} \in(0, r)$. One obtains this by comparing the $j$ th derivative of $h=\mathcal{P}+\mathcal{R}$ with the $(2 n-j)$ th order Maclaurin expansion (with Lagrange remainder) of $h^{(j)}$. The polynomial pieces are the same, while the remainder pieces give the two sides of the equality. Combining this identity with Eq. [15), we obtain

$$
\triangle_{d}^{n} \mathcal{R}(r)=r \sum_{j=1}^{2 n} b_{j} h^{(2 n+1)}\left(\xi_{j}\right)
$$

where the $b_{j}$ are ( $n$ - and $d$-dependent) constants. This shows that $\left|r^{k} \triangle_{d}^{n} \mathcal{R}(r)\right|$ is bounded by an $(n$ - and $d$-dependent) constant times $\epsilon$ for $|r| \leq 1$, so $\left|r^{k} \triangle_{d}^{n} h(r)\right|$ is, as well, proving the desired result, and hence the theorem.

Remark. The restrictions on $f$ and $d$ in the statement of the theorem are surely not optimal: Bochner's proof of the basic summation formula holds for $d \in \mathbb{R}, d>0$, and $f$ of only finite differentiability (see Theorem 9 in [5]), and there is numerical evidence that the given result holds for $d \in \mathbb{C}, \operatorname{Re} d>0$ and significantly less smooth $f$ [e.g., $f(r)=e^{-|r|^{3}}$, which is not covered by Bochner's results]. (The evidence also extends to the generalization given in Theorem 2 and is provided by a MATHEMATICA notebook, available online.9) While one could use a slightly larger function space than $\mathscr{S}^{\mathrm{E}}(\mathbb{R})$ without any change to the proof-the proof does not need control over $\|f-g\|_{n, m}$ for all $n$ and $m$-we did not investigate this in any detail: The resulting function space would still require a fair amount of differentiability (while we have numerical evidence that the formula remains true for at least some functions with a cusp at the origin), and faster decay than the standard Poisson summation formula (see, e.g., Corollary 2.6 in Chap. VII of Stein and Weiss [37]). Moreover, the closure of the family of Gaussians in this less restrictive topology would almost surely be more recondite than $\mathscr{S}^{\mathrm{E}}(\mathbb{R})$.

\section{Generalization of Theorem 1}

Since there are other families of lattices with dimensionally continued theta series besides $\mathbb{Z}^{d}$ (e.g., the root lattice $D^{d}$ mentioned in Sec. 2.1 , it is reasonable to expect that Theorem 1 can be generalized by replacing $\Theta$ with some more general function $\Upsilon$, which we shall term a generalized theta series. It is not clear how to construct the most general such $\Upsilon 19$ However, the template provided by the theta series of other standard lattices (e.g., the ones given in Chap. 4 of Conway and Sloane [10]) allows us to construct a reasonably general family of generalized theta series out of finite linear combinations of products of the three Jacobi theta functions $\left(\vartheta_{2}, \vartheta_{3}\right.$, and $\left.\vartheta_{4}\right)$ given in Eq. (3). This family will contain all of the theta series of lattices and shifted lattices given in Chap. 4 of Conway and Sloane [10], except for the general form of the theta series of the root lattice $A^{d}$ and its translates. In fact, theorems in Conway and Sloane (Theorems 7, 15, and 17 in Chap. 7 and Theorem 5 in Chap. 8) show that the theta series of large classes of lattices can be written in such a form. However, the family of $\Upsilon_{\mathrm{s}}$ is considerably more general, since one only requires $\lambda_{m}, \rho_{m}, \sigma_{m} \in \mathbb{N}_{0}$ to reproduce the theta series in Conway and Sloane, while here they can be arbitrary nonnegative real numbers. The $\Upsilon_{s}$ also contain several families of modular forms, as shown by Rankin [31], and concisely stated in Theorem 12 of Pache [30].

Explicitly, we make the following

Definition. A generalized theta series is a finite linear combinations of $\Upsilon_{d} \mathrm{~s}$ of the form

$$
\Upsilon_{d}(q):=\prod_{m=1}^{\mathcal{M}} \vartheta_{2}^{\lambda_{m}}\left(q^{s_{m}}\right) \vartheta_{3}^{\rho_{m}}\left(q^{t_{m}}\right) \vartheta_{4}^{\sigma_{m}}\left(q^{u_{m}}\right)
$$

with $\lambda_{m}, \rho_{m}, \sigma_{m} \geq 0, \sum_{m=1}^{\mathcal{M}}\left(\lambda_{m}+\rho_{m}+\sigma_{m}\right)=d$, and $s_{m}, t_{m}, u_{m} \in \mathbb{Q}_{+}$.

We thus have

$$
\Upsilon_{d}^{*}(q)=\prod_{m=1}^{\mathcal{M}} \frac{\vartheta_{2}^{\sigma_{m}}\left(q^{1 / u_{m}}\right) \vartheta_{3}^{\rho_{m}}\left(q^{1 / t_{m}}\right) \vartheta_{4}^{\lambda_{m}}\left(q^{1 / s_{m}}\right)}{\sqrt{s_{m}^{\lambda_{m}} t_{m}^{\rho_{m}} u_{m}^{\sigma_{m}}}}
$$

\footnotetext{
${ }^{9}$ The notebook is available at http://gravity.psu.edu/ nathanjm/Dim_cont_PSF_test.nb

${ }^{10}$ But note that Ryavec characterizes all admissible $\Upsilon_{\mathrm{s}}$ (under certain assumptions) for $d=1$ in [33]. We also call attention to the work of Córdoba [11] 12], who shows that in integer dimensions, large classes of generalized Poisson summation formulae arise from the standard Poisson summation formula applied to the finite disjoint union of (integer dimensional) lattices. (Note that Lagarias makes a slight correction to the statement of Theorem 2 of [11] in Theorem 3.7 of [23].)
} 
which we compute using

$$
\bar{\Upsilon}^{*}(z):=(i / z)^{d / 2} \bar{\Upsilon}(-1 / z)
$$

Nota bene: The definition of $\Upsilon^{*}$ is just the dimensionally continued Jacobi transformation [Eq. (44] of $\Upsilon$ with the factor of $\sqrt{\operatorname{det} \Upsilon}$ omitted. (We leave off this factor, since it would just cancel against the one present in the standard Poisson summation formula [cf. Eq. [1]].)

We now need to show that the generalized theta series have the appropriate properties to allow us to copy the proof of Theorem 1 almost verbatim. Specifically, we need the following

Proposition. If $\Upsilon$ denotes any of the $\Upsilon_{d} s$ or $\Upsilon_{d}^{*} s$ defined above, we can write

$$
\Upsilon(q)=\sum_{l=0}^{\infty} N_{l} q^{A_{l}}
$$

where

1. $A_{l+1}>A_{l}, A_{0} \geq 0$.

2. $\sum_{l=1}^{\infty} A_{l}^{-2}<\infty$.

3. There exists $L \in \mathbb{N}$ and $C, n>0$ such that $\left|N_{l}\right| \leq C A_{l}^{n}$ for all $l \geq L$.

4. The series converges inside the unit disk.

Proof. First note that we can apply the same arguments to $\Upsilon_{d}^{*}$ as to $\Upsilon_{d}$, so we can restrict our attention to the former, without loss of generality. Now, we then have

$$
A_{l}=(l+\mathcal{A}) / V, \quad \mathcal{A}:=\sum_{m=1}^{\mathcal{M}} V \lambda_{m} s_{m} / 4
$$

where $V$ is the least common denominator of $s_{m}, t_{m}$, and $u_{m}$ (for all $m$ ). [We have the additive constant $\mathcal{A}$ due to the overall factor of $q^{1 / 4}$ in $\vartheta_{2}(q)$.] Thus the first required and second required properties (positivity and monotonicity of the $A_{l}$ and convergence of the series whose terms are $A_{l}^{-2}$ ) are obviously true.

For the third property (polynomial boundedness of the $N_{l}$ ), we use the same Cauchy's integral formula argument used in Sec. 5.1] (The analyticity established below shows that Cauchy's theorem is still applicable here.) Here $N_{l}$ is given by the $l$ th term in the Maclaurin expansion of $\Upsilon_{d}\left(q^{V}\right) / q^{\mathcal{A}}$, so we have

$$
\begin{aligned}
\left|N_{l}\right| & =\left|\frac{1}{2 \pi i} \int_{\mathrm{C}_{R}} \prod_{m=1}^{\mathcal{M}} \frac{\vartheta_{2}^{\lambda_{m}}\left(z^{V s_{m}}\right) \vartheta_{3}^{\rho_{m}}\left(z^{V t_{m}}\right) \vartheta_{4}^{\sigma_{m}}\left(z^{V u_{m}}\right)}{z^{V \lambda_{m} s_{m} / 4} z^{l+1}} d z\right| \\
& \leq 2^{d} \prod_{m=1}^{\mathcal{M}} \frac{1}{R^{l}\left(1-R^{V s_{m}}\right)^{\lambda_{m}}\left(1-R^{V t_{m}}\right)^{\rho_{m}}\left(1-R^{V u_{m}}\right)^{\sigma_{m}}} \leq \frac{2^{d}}{R^{l}(1-R)^{d}},
\end{aligned}
$$

where $C_{R}$ is the same contour used previously. We have used the geometric series to obtain the bound $|\tau(q)| \leq$ $2 /(1-|q|)$, where $\tau(q)$ is any of $\vartheta_{2}(q) / q^{1 / 4}, \vartheta_{3}(q)$, or $\vartheta_{4}(q)$. Additionally, we have used the fact that $\kappa \geq 1$, where $\kappa$ is any of $V s_{m}, V t_{m}$, or $V u_{m}$, so $\left|1-R^{\kappa}\right| \geq 1-R$, since $R \in(0,1)$. We also recalled that $\lambda_{m}, \rho_{m}, \sigma_{m} \geq 0$ and $\sum_{m=1}^{\mathcal{M}}\left(\lambda_{m}+\rho_{m}+\sigma_{m}\right)=d$. Since there is an $R \in(0,1)$ such that $2^{d} /\left[R^{l}(1-R)^{d}\right] \leq C_{d} l^{d}$ (for $l \geq 1$ ), as was shown in Sec.5.1, we are done.

The fourth property (convergence of the $q$-series in the unit disk) follows from the analyticity and lack of zeros of the theta functions inside the unit disk, as in the argument given below Eq. 112]. [Note that here we consider $\vartheta_{2}(q) / q^{1 / 4}$, not $\vartheta_{2}(q)$ itself.] Specifically, $\Upsilon\left(q^{V}\right) / q^{\mathcal{A}}$ is an analytic function of $q$ inside the unit disk; the lack of zeros can be seen from the infinite product representations of $\vartheta_{2}$ and $\vartheta_{4}$ given, e.g., in Eqs. (34) and (36) in Chap. 4 of Conway and Sloane [10].

The dimensionally continued summation formula for generalized theta series thus takes the form of the following

Theorem 2. Let $\Upsilon$ be a generalized theta series (as defined above), with power series coefficients and powers $N_{l}$ and $A_{l}$, i.e.,

$$
\Upsilon(q)=\sum_{l=0}^{\infty} N_{l} q^{A_{l}}
$$


Let $N_{l}^{*}$ and $A_{l}^{*}$ be the corresponding quantities for $\Upsilon$ 's dual, $\Upsilon^{*}$ [computed using Eq. [17]]. Then, for any $f \in \mathscr{S}^{\mathrm{E}}$, we have the summation formula

$$
\sum_{l=0}^{\infty} N_{l} f\left(\sqrt{A_{l}}\right)=\sum_{l=0}^{\infty} N_{l}^{*} \hat{f}\left(\sqrt{A_{l}^{*}}\right)
$$

where we compute $\hat{f}$ using Eq. (6) (with the dimension parameter $d$ associated with $\Upsilon$ ).

Remark. In general, all one requires of the $\Upsilon_{s}$ used in this summation formula is that they and their Jacobi transformations have sufficiently well-behaved power series. (This is satisfied by all functions of weight, in the terminology of Pache [30]—-see Pache's Definition 11.) However, it is unclear whether any such functions exist besides the generalized theta series we have defined above, so we have not given the theorem in a more general form.

Proof. The proof is almost the same as that for Theorem 1 (replacing $\Theta$ by $\Upsilon$, and noting that we can no longer appeal to the standard Poisson summation formula for $d=1$, so we simply exclude that case). Most of the work has been done in the proof of the Proposition; the only new part is checking that $\sum_{l=0}^{\infty}\left|N_{l} h\left(\sqrt{A_{l}}\right)\right| \rightarrow 0$ as $\epsilon \rightarrow 0$ if $h$ is $\epsilon$-close to 0 in the Schwartz space topology [and similarly for $\sum_{l=0}^{\infty}\left|N_{l}^{*} h\left(\sqrt{A_{l}^{*}}\right)\right|$ ]. To do this, we simply note that we have $\left|N_{l} h\left(\sqrt{A_{l}}\right)\right| \leq C A_{l}^{n}\left|h\left(\sqrt{A_{l}}\right)\right|$, by polynomial boundedness of the $N_{l}$, and that $x^{2 n+4}|h(x)| \leq \epsilon$ $\forall x \in \mathbb{R} \Rightarrow A_{l}^{n}\left|h\left(\sqrt{A_{l}}\right)\right| \leq \epsilon / A_{l}^{2}$ [cf. the discussion at the end of Sec. 5.1], from which the desired result follows immediately. (The same argument holds for the starred quantities, since they have the same properties as the unstarred quantities.)

Remark. This theorem can likely be interpreted as a trace formula for the dimensionally continued, spherically symmetric Laplacian [Eq. [14)], since the kernel of the dimensionally continued Fourier transform is an eigenfunction of this operator (see Lemma 4). See, e.g., Sec. 1.3 (particularly Theorem 1.3) of Uribe [39] for a presentation of the standard Poisson summation formula for an integer dimension lattice as a trace formula for the Laplacian.

Remark. This result shows that one can apply this extended Poisson summation formula to lattice-like objects whose theta series have coefficients of both signs, so they do not exist as a lattice, even though $d \in \mathbb{N}$ : For a trivial example, consider $d=2$ and $\Phi(q)=\vartheta_{4}^{2}(q)=1-4 q+4 q^{2}+\cdots$. Of course, this is in some sense too trivial, since one can write $\vartheta_{4}^{2}=2 \Theta_{D^{2}}-\Theta_{\mathbb{Z}^{2}}$, and then apply the standard Poisson summation formula to each of those lattices to establish the result in this case (cf. the discussion in Córdoba [12]). However, in more complicated higher-dimensional cases, it will likely not be clear how to construct the lattice(s) associated with the theta series (if they indeed exist). Indeed, Jenkins and Rouse [19] have very recently shown that for weights higher than 81632 , all the modular forms of a certain type have coefficients of both signs. (This general property was first shown in less specific, much earlier work by Mallows, Odlyzko, and Sloane [25].)

\section{OUTLOOK}

While Theorem 2 encompasses quite a large family of summation formulae, there still remains wide latitude for further generalizations (even excluding the various possibilities for weakening certain of the hypotheses mentioned after Theorem 1). The most sweeping generalization would likely be to replace the dimensionally continued Fourier transform with some more general family of integral transforms, with the possibility of a subsequent enlargement of the transformation properties required of the generalized theta series. Here one could follow the work of Kubota [22] and Unterberger [38] in integer dimensions. But even if one retains the dimensionally continued Fourier transform, one can still likely obtain summation formulae from more general classes of generalized theta series than we have considered. In particular, it would be interesting to obtain a dimensionally continued version of the quasicrystal summation formula given as Theorem 2.9 in Lagarias [23]. Here the calculations of the central shelling for certain quasicrystals in, e.g., [3, 27, 28, 29] could be relevant. Additionally, since the coefficients of standard theta series give the representation numbers for lattices, it is possible that our results could be applicable to generalizations of representation number problems: See, e.g., [16] for a review of standard results on representation numbers.

\section{ACKNOWLEDGEMENTS}

It is our pleasure to thank George Andrews, Jingzhi Tie, and Shuzhou Wang for encouragement and useful comments, John Roe for mentioning an alternative proof of the density result, Artur Tšobanjan for helpful remarks about the exposition, and the anonymous referee for various perceptive comments and suggestions. We also thank Ben Owen 
for comments on the manuscript and for suggesting the physics problem that sparked these investigations. This work was supported by NSF grants PHY-0555628 and PHY-0855589, the Eberly research funds of Penn State, and the DFG SFB/Transregio 7.

\section{REFERENCES}

1. G. E. Andrews, R. Askey, and R. Roy, Special Functions, Encyclopedia of Mathematics and Its Applications, vol. 71, Cambridge University Press, Cambridge, 1999.

2. M. Baake, D. Frettlöh, and U. Grimm, A radial analogue of Poisson's summation formula with applications to powder diffraction and pinwheel patterns, J. Geom. Phys. 57 (2007), 1331-1343.

3. M. Baake and U. Grimm, A note on shelling, Discrete Comput. Geom. 30 (2003), 573-589.

4. R. Bellman, A Brief Introduction to Theta Functions, Holt, Rinehart and Winston, New York, 1961.

5. S. Bochner, Some properties of modular relations, Ann. Math. 53 (1951), 332-363.

6. Harmonic Analysis and the Theory of Probability, University of California Press, Berkeley, 1955.

7. H. Cohen, Number Theory, Volume II: Analytic and Modern Tools, Springer, New York, 2007.

8. R. Contino and A. Gambassi, On dimensional regularization of sums, J. Math. Phys. 44 (2003), 570-587.

9. J. B. Conway, A Course in Functional Analysis, second ed., Springer, New York, 1990.

10. J. H. Conway and N. J. A. Sloane, Sphere Packings, Lattices and Groups, third ed., Springer, New York, 1999.

11. A. Córdoba, La formule sommatoire de Poisson, C. R. Acad. Sci., Paris, Sér. I 306 (1988), 373-376.

12. Dirac combs, Lett. Math. Phys. 17 (1989), 191-196.

13. W. L. Ferrar, Summation formulae and their relation to Dirichlet's series II, Compos. Math. 4 (1937), $394-405$.

14. A. P. Guinand, Integral modular forms and summation formulae, Proc. Cambridge Phil. Soc. 43 (1947), $127-129$.

15. Concordance and the harmonic analysis of sequences, Acta. Math. 101 (1959), 235-271.

16. J. Hanke, Some recent results about (ternary) quadratic forms, Number Theory (H. Kisilevsky and E. Z. Goren, eds.), CRM Proc. Lecture Notes, vol. 36, American Mathematical Society, Providence, RI, 2004, pp. 147-164.

17. M. N. Huxley, Area, Lattice Points, and Exponential Sums, Oxford University Press, New York, 1996.

18. H. Iwaniec and E. Kowalski, Analytic Number Theory, American Mathematical Society, Providence, RI, 2004.

19. P. Jenkins and J. Rouse, Bounds for coefficients of cusp forms and extremal lattices, Bull. London Math. Soc. 43 (2011), $927-938$.

20. R. E. Johnson and S. Ranganathan, Generalized approach to Ewald sums, Phys. Rev. E 75 (2007), 056706.

21. N. K. Johnson-McDaniel and B. J. Owen, Shear modulus of the hadron-quark mixed phase, arXiv:1110.4650 [astro-ph.SR].

22. T. Kubota, On an analogy to the Poisson summation formula for generalized Fourier transformation, J. Reine Angew. Math. $268 / 269$ (1974), $180-189$.

23. J. C. Lagarias, Mathematical quasicrystals and the problem of diffraction, Directions in Mathematical Quasicrystals (M. Baake and R. V. Moody, eds.), CRM Monograph Series, vol. 13, American Mathematical Society, Providence, RI, 2000, pp. 61-93.

24. S. Lang, Real and Functional Analysis, third ed., Springer, New York, 1993.

25. C. L. Mallows, A. M. Odlyzko, and N. J. A. Sloane, Upper bounds for modular forms, lattices, and codes, J. Algebra 36 (1975), $68-76$.

26. S. D. Miller and W. Schmid, Summation formulas, from Poisson and Voronoi to the present, Noncommutative Harmonic Analysis: In honor of Jacques Carmona (P. Delorme and M. Vergne, eds.), Birkhäuser, Boston, 2004, pp. 419-440.

27. R. V. Moody and J. Patera, Quasicrystals and icosians, J. Phys. A, Math. Gen. 26 (1993), 2829-2853.

28. R. V. Moody and A. Weiss, On shelling E $E_{8}$ quasicrystals, J. Number Theory 47 (1994), 405-412.

29. J. Morita and K. Sakamoto, Octagonal quasicrystals and a formula for shelling, J. Phys. A, Math. Gen. 31 (1998), $9321-9325$.

30. C. Pache, Shells of selfdual lattices viewed as spherical designs, Int. J. Algebra Comput. 15 (2005), $1085-1127$.

31. R. A. Rankin, Modular Forms and Functions, Cambridge University Press, Cambridge, 1977.

32. M. Reed and B. Simon, Methods of Modern Mathematical Physics I: Functional Analysis, rev. and enl. ed., Academic Press, New York, 1980.

33. C. Ryavec, The Poisson summation formula, Aequationes Math. 21 (1980), 246-250.

34. L. Schwartz, Théorie des Distributions, third ed., Hermann, Paris, 1998.

35. B. Simon, Distributions and their Hermite expansions, J. Math. Phys. 12 (1971), 140-148.

36. A. Sklar, On Some Exact Formulae in Analytic Number Theory, Report of the Institute in the Theory of Numbers (D. C. B. Marsh, ed.), American Mathematical Society, Boulder, CO, 1959, pp. 104-110.

37. E. M. Stein and G. Weiss, Introduction to Fourier Analysis on Euclidean Spaces, Princeton University Press, Princeton, 1971.

38. A. Unterberger, A spectral analysis of automorphic distributions and Poisson summation formulas, Ann. Inst. Fourier 54 (2004), $1151-1196$.

39. A. Uribe, Trace formulae, First Summer School in Analysis and Mathematical Physics, Cuernavaca, Mexico (S. Pérez-Esteva and C. VillegasBlas, eds.), Contemp. Math., vol. 260, American Mathematical Society, 2000, pp. 61-90.

40. E. T. Whittaker and G. N. Watson, A Course of Modern Analysis, fourth ed., Cambridge University Press, Cambridge, 1927.

41. Wolfram Research, The Wolfram Functions Site, 2011, http://functions.wolfram.com.

Institute for Gravitation and the Cosmos, Center for Particle and Gravitational Astrophysics, Department of Physics, The Pennsylvania State University, University Park, PA 16802, USA

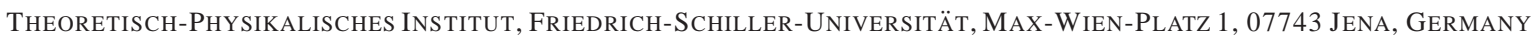

E-mail address: nathan-kieran.johnson-mcdaniel@uni-jena.de 